\title{
Critical acceleration of finite-temperature SU(2) gauge simulations
}

\author{
Radel Ben-Av \\ Department of Nuclear Physics, Weizmann Institute of Science, 76100 Rehovot, Israel \\ Hans Gerd Evertz \\ Supercomputer Computations Research Institute, Florida State University, Tallahassee, Florida 32306 \\ Mihail Marcu \\ School of Physics and Astronomy, Raymond and Beverly Sackler Faculty of Exact Sciences, \\ Tel Aviv University, 69978 Tel Aviv, Israel \\ and II. Institut für Theoretische Physik, Universität Hamburg, Luruper Chaussee 149, D-2000 Hamburg 50, Germany \\ Sorin Solomon \\ Racah Institute of Physics, Hebrew University, 91904 Jerusalem, Israel
}

(Received 2 May 1991)

\begin{abstract}
We present a cluster algorithm that strongly reduces critical slowing down for the SU(2) gauge theory on one time slice. The idea that underlies the new algorithm is to perform efficient flips for the signs of Polyakov loops. Ergodicity is ensured by combining it with a standard local algorithm. We show how to quantify critical slowing down for such a mixed algorithm. At the finite-temperature transition, the dynamical critical exponent $z$ is $\approx 0.5$, whereas for the purely local algorithm $z \approx 2$.
\end{abstract}

\section{INTRODUCTION}

Finding an efficient simulation algorithm for pure gauge theories is one of the main challenges in the field of Monte Carlo simulations. In this paper we report on a new cluster algorithm for the finite-temperature SU(2) pure gauge theory in $3+1$ dimensions [1]. Our method is based on efficient updates of the signs of the Polyakov loops, and works in the case of an $L^{3} \times N_{t}$ lattice for $N_{t}=1$. This is the first time that a cluster method was successfully applied for a continuous-group gauge model (the only other case of a successful cluster algorithm for a gauge theory is in the case of the discrete group $Z_{2}$ [2]).

Although the $N_{t}=1$ case is not really the physically relevant one, we decided to study it in detail for a variety of reasons. First, it is a test of whether our whole approach for obtaining a cluster algorithm for the study of the finite-temperature transition is meaningful. It is not $a$ priori clear that speeding up the Polyakov-loop updates alone will suffice to overcome critical slowing down. Second, there is little experience in the analysis of the autocorrelations in a situation where a nonergodic cluster algorithm is combined with an ergodic local algorithm. Last but not least, at the present stage any progress at all in cluster algorithms for gauge theories may be relevant.

Ultimately, we believe that only an accelerated algorithm will enable us to settle the question of whether the finite-temperature phase transition is driven by the Polyakov loops or if other phenomena, such as monopole condensation [3], are relevant in the large- $N_{t}$ regime. [1]

The SU(2) pure gauge theory is defined by the action

$$
S=-\beta \sum_{p} \frac{1}{2} \operatorname{Tr} U(p),
$$

where $U_{x, \mu}$ are $2 \times 2 \mathrm{SU}(2)$ matrices, $x$ is a site on an
$L^{3} \times N$, lattice, $x, \mu$ is a link of this lattice, and $U(p)$ is the product of link variables along the boundary of an elementary plaquette $p$. Periodic boundary conditions are assumed.

Speeded-up algorithms may be obtained by directly acting on the relevant degrees of freedom. At small values of $N_{t}$, it is generally accepted that the phase transition is driven by the Polyakov loops [1]. A Polyakov loop at the spatial point $\mathbf{x}$ is defined by (boldface quantities always denote spatial, three-dimensional, objects)

$$
P_{\mathrm{x}}:=\frac{1}{2} \operatorname{Tr} \prod_{t=1}^{N_{t}} U_{(\mathrm{x}, t), 0}
$$

Therefore, we attempted to devise cluster algorithms [4-6] that significantly speed up the Polyakov-loop pseudodynamics. As the basis for such algorithms we took involutive operations ("flips") [7-9] on appropriately chosen degrees of freedom. Following [7] we call these degrees of freedom embedded Ising variables. In order to ensure ergodicity, the cluster updates have to be supplemented by conventional local Metropolis [10] or heatbath [11] updates.

\section{THE CLUSTER METHOD}

The embedded Ising variables for our cluster algorithm are the signs of the Polyakov loops. One way to have the Polyakov loops among the degrees of freedom in terms of which the model is written is to go to the temporal gauge (" $A_{0}=0$ "). Now, in order to obtain a successful algorithm that does overcome critical slowing down, the embedded Ising model should not be frustrated [4-6]. For the signs of the Polyakov loops, however, we obtain, for general $N_{t}$, an effective interaction that is frustrated. Furthermore, we also obtain an undesired magnetic field 
term, whose fluctuating sign and strength depends on the other degrees of freedom.

For the case of $N_{t}=1$ an important simplification occurs: there is no frustration and no magnetic field term. For the timelike plaquette $p$ that connects the spatial points $\mathbf{x}$ and $\mathbf{x}+\hat{j}$ ( $\hat{j}$ is a lattice spacing vector in the spatial $j$ direction),

$$
\frac{1}{2} \operatorname{Tr} U(p)=P_{\mathbf{x}} P_{\mathbf{x}+\hat{j}}+\text { const },
$$

where the constant does not depend on the signs of $P_{\mathrm{x}}$ and $P_{\mathrm{x}+\hat{j}}$. This corresponds to a ferromagnetic interaction of the embedded Ising variables, the effective coupling being $\beta\left|P_{\mathrm{x}} P_{\mathrm{x}+\hat{j}}\right|$.

In this situation our cluster algorithm is defined as follows. First we delete or freeze the links $x, j$ (we use the terminology of [12] here). The deletion probability for a link is

$$
P_{\mathrm{del}}(\mathrm{x}, j)=\exp \left[-\beta\left(P_{\mathrm{x}} P_{\mathrm{x}+\hat{j}}+\left|P_{\mathrm{x}} P_{\mathrm{x}+\hat{j}}\right|\right)\right] .
$$

The freezing probability is $1-P_{\mathrm{del}}(\mathbf{x}, j)$. Two sites are connected if they are at the end points of a frozen link. A cluster is a connected set of sites (thus, of course, all links from a site in a given cluster to one outside it are deleted; on the other hand, two nearest neighbors that are part of the same cluster are not necessarily connected by a frozen link). In order to update the configuration we use the single-cluster algorithm [5,8]: a cluster is "grown" around a randomly chosen site (the seed) and flipped with probability one. A flip means the operation $P_{\mathbf{x}} \rightarrow-P_{\mathrm{x}}$ for all Polyakov loops in the cluster. Note that we flip only the diagonal part of timelike links $U_{x, 0}=P_{\mathbf{x}} \mathbf{1}+i \mathbf{u}_{\mathbf{x}} \cdot \sigma$; flipping $U_{x, 0} \rightarrow-U_{x, 0}$ would not eliminate frustration.

We supplemented the cluster update with a local update of all degrees of freedom. For the timelike links we used the heat-bath procedure of [11]. Because of the $N_{t}=1$ condition, we had to use a standard Metropolis procedure to update the spatial links.

\section{STRATEGY FOR THE SIMULATION}

First we determined the exact location of the phase transition from a standard finite-size analysis. We obtained $\beta_{c}=0.8730(2)$ and critical exponents compatible with those of the three-dimensional Ising model [13]. We shall not give details of this analysis here, since, up to the exact location of $\beta_{c}$, the results are what we expected in accordance with the assumption of dynamical universality. We shall rather concentrate on presenting our results for the autocorrelation times at the critical point, i.e., on the quantitative study of critical slowing down for the new algorithm.

Since we deal with a mixture of two algorithms (cluster and local), let us start by defining one time unit in the Markov chain as one local sweep followed by $n$ cluster flips. Let us further define the ratio $R$,

$$
R:=n \frac{\langle\text { cluster size }\rangle}{L^{3}}
$$

of the work performed in the cluster part to that performed in the local part. Let $\tau(L, R)$ be the exponential autocorrelation time at the critical point $\beta_{c}$ (i.e., the slowest mode of the update procedure) [6]. The dynamical critical exponent $z$ is usually defined by the relation $\tau \propto L^{z}[4-6,14]$. In our case we can define an $R$-dependent exponent $z(R)$ if, for a fixed value of $R$, the relation

$$
\tau(L, R) \propto L^{z(R)}
$$

is fulfilled. Studying the dependence of $\tau(L, R)$ on $R$ is also very interesting since little is known about the analysis of critical slowing down for a mixed algorithm. Notice that for a fixed $L$, the limit $R \rightarrow \infty$ is the "idealized embedding" discussed in [6].

For a sufficiently accurate determination of $\tau$, the runs at $\beta_{c}$ were very long, between 30000 and 100000 time units (for the study of the static critical properties alone, much shorter runs suffice, since our algorithm is very efficient). We measured a large number of observables, including space- and timelike plaquettes, absolute value of the lattice-averaged Polyakov loop $\left|L^{-3} \Sigma_{\mathrm{x}} P_{\mathrm{x}}\right|$, and spacelike Wilson loops. We also defined and measured improved observables $[5,8,9]$, e.g., for correlations between Polyakov loops (details on the improved observables will be presented elsewhere). Exponential autocorrelation times were consistent for all observables that did show slowing down at all (there were no visible autocorrelations for spacelike plaquettes and Wilson loops).

We took great care to perform a thorough error analysis. We took into account the covariances of autocorrelations at different time distances. We checked all results for stability by doing a complete jackknife analysis. We were also careful to verify whether the plots of the autocorrelation functions do show a clear exponential decay - such a check had turned out to be crucial during a concurrent cluster investigation [15].

\section{RESULTS}

First and utmost, the new algorithm spectacularly reduces critical slowing down. To exemplify this, we compare in Fig. 1 two autocorrelation functions for a $32^{3} \times 1$ lattice at $\beta=\beta_{c}$, one for a purely local update, the other for $R=0.18$ (for the definition of autocorrelation functions see [4-6]). Although in the latter case for each local

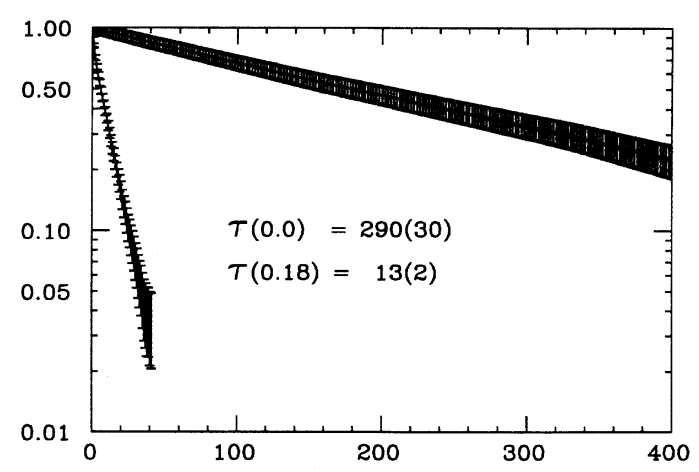

FIG. 1. Autocorrelation functions at $\boldsymbol{\beta}_{c}$ for the Polyakov loop on a $32^{3} \times 1$ lattice, without clusters, and with $R=0.18$. 


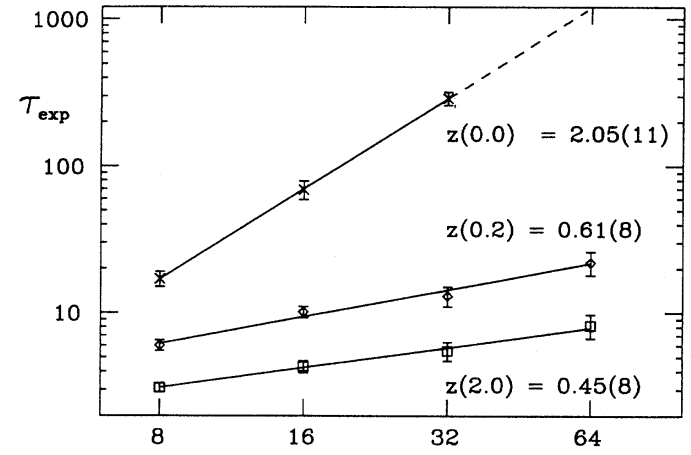

FIG. 2. Log-log plot of autocorrelation times of Polyakov loops at $\beta_{c}$ vs $L$, for $R=0, R \approx 0.2$, and $R \approx 2$.

sweep only $18 \%$ of the lattice sites are changed by cluster updates, the speedup is tremendous. The $64^{3} \times 1$ lattice would have been totally unaccessible without cluster updates.

As expected, $z \approx 2$ for the purely local algorithm [14]: we obtain $z(0)=2.05(11)$ from runs for $L \leq 32$ (see Fig. 2 ). For two very different values of $R, R \approx 0.2$ and 2 , we performed measurements of $z(R)$ up to $L=64$ (the fact that $R$ was not exactly 0.2 or 2 but varied slightly with $L$, e.g., $R=0.18$ instead of $R=2$ for $L=32$, does not affect the results within our statistical errors). The relation (6) was well obeyed, as seen again from Fig. 2. The value of $z(R)$ is drastically reduced to $z(0.2)=0.61(8)$, and to $z(2)=0.45(8)$.

In order to study further the $R$ dependence of the autocorrelation time, we performed additional runs for $L=8$, 16, and 32. The results are collected in Fig. 3. At fixed $L$, $\tau(L, R)$ shows a very fast initial dropoff, roughly like $1 / R$. Then, as $R \rightarrow \infty$, it goes to a constant asymptotic value $\tau_{\infty}(L)$, which corresponds to the "idealized algorithm" of [6]. Notice that Fig. 3 suggests $R=2$ to already be almost asymptotic.

In our implementation, the CPU time for the cluster and for the local update was roughly the same (in our case the local part vectorized completely). Thus the CPU time necessary to perform one time unit was roughly proportional to $1+R$. An analysis of the data presented in Fig. 3 shows that $R \approx 0.5$ will be the most effective algorithm: for a given value of $L$, the quantity $(1+R) \tau(L, R)$, i.e., the autocorrelation time in CPU units, is minimized at $R \approx 0.5$. If, e.g., the local part were not vectorized, a smaller value of $R$ would give optimal performance.

It is perhaps of interest to note that all results of Fig. 3 are well described by the phenomenological formula

$\tau(L, R)=\tau_{\infty}(L)+\left(\frac{R}{\tau_{\mathrm{cl}}(L)}+\frac{1}{\tau_{\mathrm{loc}}(L)-\tau_{\infty}(L)}\right)^{-1}$

where $\tau_{\infty}(L) \propto L^{z_{\infty}}$ is the autocorrelation time of the idealized $R \rightarrow \infty$ algorithm, $\tau_{\text {loc }} \propto L^{z_{\text {loc }}}$ is the autocorrela-

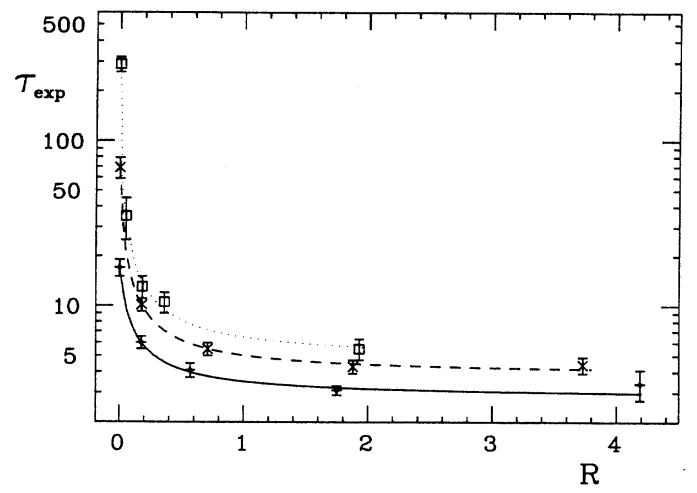

FIG. 3. Autocorrelation times vs $R$ for $L=8,16$, and 32. The curves are fits according to (7).

tion time of the purely local algorithm, and $\tau_{\mathrm{cl}}(L)$ may be interpreted as the autocorrelation time for cluster updates of the effective Ising models. The best fit for the various dynamical exponents of (7) is $z_{\infty}=0.42(10), \quad z_{\text {loc }}$ $=2.05(11)$, and $z_{\mathrm{cl}}=0.64(19)$.

Notice that $z_{\infty} \approx z(2)$, in accordance with the previous observation that at $R \approx 2, \tau(L, R)$ is already almost asymptotic in $R$. These values for the dynamical critical exponent are consistent with the value obtained by [16] for the three-dimensional Ising model with a single-cluster algorithm.

In order to check the form (7) with good accuracy, a lot of expensive runs at small values of $R$ are needed. However, a good theoretical understanding of autocorrelations of a combination of two algorithms should be achieved first.

\section{CONCLUSIONS}

We have shown how to implement a cluster algorithm for the $S U(2)$ lattice gauge model with $N_{t}=1$. For the first time ever in the framework of continuous-group gauge theories, critical slowing down was drastically reduced. This is due to a procedure that performs largescale fluctuations for the relevant degrees of freedom. As a by-product of our investigation, we gained insight into the behavior of autocorrelations for an algorithm that combines two different update procedures.

We believe that similar methods may evolve into a cluster method for investigating the finite-temperature transition of pure gauge theories.

\section{ACKNOWLEDGMENTS}

We thank Alan Sokal for valuable discussions. At various stages, the kind hospitality of the Weizmann Institute and the Hebrew University allowed us to come together. We thank the HLRZ Jülich for generous allocation of Cray computer time. This work was supported in part by the Deutsche Forschungsgemeinschaft, the GermanIsraeli Foundation, and the Israeli Academy of Sciences. 
[1] See, e.g., B. Svetitsky and L. G. Yaffe, Nucl. Phys. B210, 423 (1982); C. Borgs and E. Seiler, ibid. B215, 125 (1983); Commun. Math. Phys. 91, 329 (1983); A. Ukawa, in Lattice '89, Proceedings of the International Symposium, Capri, Italy, 1989, edited by R. Petronzio et al. [Nucl. Phys. B (Proc. Suppl.) 17, 118 (1990)].

[2] R. Ben-Av, D. Kandel, E. Katznelson, P. Lauwers, and S. Solomon, J. Stat. Phys. 58, 125 (1990).

[3] G. 't Hooft, Nucl. Phys. B138, 1 (1978).

[4] R. H. Swendsen and J. S. Wang, Phys. Rev. Lett. 58, 86 (1987).

[5] U. Wolff, in Lattice '89 (Ref. [1]), p. 93.

[6] A. Sokal, in Cours de Troisième Cycle de la Physique en Suisse Romande, Lausanne, Switzerland, 1989 (unpublished); in Lattice '90, Proceedings of the Conference, Tallahassee, Florida, 1990, edited by U. M. Heller, A. D. Kennedy, and S. Sanielevici [Nucl. Phys. B (Proc. Suppl.) 20 (1990)].

[7] R. C. Brower and P. Tamayo. Phys. Rev. Lett. 62, 1087
(1989).

[8] U. Wolff, Phys. Rev. Lett. 62, 361 (1989); Nucl. Phys. B322, 759 (1989); Phys. Lett. B 228, 379 (1989).

[9] M. Hasenbusch, Nucl. Phys. B333, 581 (1990); M. Hasenbusch and S. Meyer, Phys. Lett. B 241, 238 (1990).

[10] N. Metropolis et al., J. Chem. Phys. 21, 1087 (1953).

[11] M. Creutz, Phys. Rev. D 21, 2308 (1980); K. Fredenhagen and M. Marcu, Phys. Lett. B 193, 486 (1987).

[12] D. Kandel et al., Phys. Rev. Lett. 60, 1591 (1988); D. Kandel, E. Domany, and A. Brandt, Phys. Rev. B 40, 330 (1989); D. Kandel and E. Domany, ibid. 43, 8539 (1991).

[13] D. Pawley et al., Phys. Rev. B 29, 4030 (1984).

[14] P. C. Hohenberg and B. I. Halperin, Rev. Mod. Phys. 49, 435 (1977).

[15] H. G. Evertz, M. Hasenbusch, M. Marcu, Klaus Pinn, and S. Solomon, Phys. Lett. B 254, 185 (1991).

[16] P. Tamayo, R. C. Brower, and W. Klein, Boston University report, 1989 (unpublished). 